\title{
Comparison between different methods of estimation of vitamin D
}

\author{
Mrinal Pal $^{1 *}$, Subinay Datta ${ }^{1}$, Amit Kumar Pradhan ${ }^{1}$, Lekha Biswas ${ }^{1}$, Joydeep Ghosh ${ }^{1}$, \\ Partha Mondal $^{1}$, Rajarshi Rahut ${ }^{1}$, Arghya Ray Chaudhuri ${ }^{1}$, Sourav Sau ${ }^{2}$, Soumi Das ${ }^{1}$ \\ ${ }^{1}$ Department of Biochemistry, Burdwan Medical College, Burdwan, India \\ ${ }^{2}$ Department of Radiotherapy, Burdwan Medical College, Burdwan, India \\ Email: ${ }^{*}$ mrinalpal77@,rediffmail.com
}

Received 3 September 2013; revised 1 October 2013; accepted 14 October 2013

Copyright (C) 2013 Mrinal Pal et al. This is an open access article distributed under the Creative Commons Attribution License, which permits unrestricted use, distribution, and reproduction in any medium, provided the original work is properly cited.

\begin{abstract}
Background: Vitamin $D$ is not only important for bone health but can also affect the development of several non-bone diseases. These findings have increased the need for determining vitamin $D$ status in a convenient and cost-effective way. In order to determine the better method for detecting vitamin $D$ status, we have estimated blood vitamin $D$ in three methods such as high-pressure liquid chromatography, chemiluminescent immunoassays and enzyme immunoassays. Method: Two hundred and sixteen subjects irrespective of age and sex were studied for blood vitamin $D$ in all the three methods over a period of 2 years. Result: The statistical analysis showed that values of vitamin $D$ obtained by chemiluminescent immunoassays $(r=0.978, p<0.001)$ were more significantly correlated with high-pressure liquid chromatography than enzyme immunoassays $(r=$ 0.970, $p<0.001)$. Conclusion: We conclude that chemiluminescent immunoassays should be used instead of enzyme immunoassay and high-pressure liquid chromatography for estimation of vitamin $D$.
\end{abstract}

Keywords: Vitamin D; High-Pressure Liquid Chromatography; Chemiluminescent Immunoassays; Enzyme Immunoassays

\section{INTRODUCTION}

Vitamin D deficiency is not only associated with osteoporosis and osteomalacia [1,2] but can also contribute to decreased muscle strength [3], cancers [4], cardiovascular disease [5], type 1 diabetes mellitus [6] and overall mortality [7]. These findings have increased the need for determining vitamin D status in a reliable way. There are

*Corresponding author. many commercially available 25-hydroxyvitamin D (25OHD) assays used for determination of vitamin D status. These include high-pressure liquid chromatography (HPLC) [8], enzyme immunoassays (EIA), and chemiluminescent immunoassays (CLIA). Determination of $25 \mathrm{OHD}$ by HPLC can be considered the gold standard method [9]. However, it is accepted that these methods are unsuitable for routine, clinical laboratory use as the method is very time consuming, requiring extensive purification steps [10]. But there is still continuing uncertainty as which method should be adopted to measure the concentration of vitamin D or should be estimated easily to be the definite way. So, the present study therefore is to investigate which method is cost-effective and reliable to analyse the vitamin D status.

\section{MATERIAL AND METHOD}

\subsection{Study Area}

The present study was conducted in the Department of Biochemistry of Burdwan Medical College, Burdwan in collaboration of Department of Biochemistry, Medical College, Kolkata and Gautam Laboratories, Kolkata, West Bengal.

\subsection{Selection of Subjects}

One thousand patients residing Burdwan district and adjoining areas were selected in this study. Patients having any concomitant infection and recent history of taking drugs that can affect the blood vitamin D concentration were not included in the study. Then two hundred and sixteen subjects were selected by a simple random method for this study. All the patients were accepted to participate by giving informed consent. The proposal of this study was approved by the Ethics Committee of Burdwan Medical College, West Bengal. 


\subsection{Collection of Samples}

After noting the age and sex, venous blood samples were collected after a 12-h overnight fasting, protected from light, centrifuged and stored at $-80^{\circ} \mathrm{C}$ until analysis.

\subsection{Parameter Assay}

Vitamin D in all samples was analyzed using three techniques HPLC, CLIA and EIA.

\subsubsection{High-Pressure Liquid Chromatography (HPLC)}

Determination of 25-hydroxyvitamin D in plasma was done by HPLC (JASCO open HPLC system; Model: LC NET II/ADC, Detector: UV-2075plus, pump LC-2080 series, Software: SF-NAV with DG-2080-53/4) using a C18 reverse phase column with gradient elution (acetonitrile/water). The HPLC unit was an integrated system with a UV-2075plus detector set at $275 \mathrm{~nm}$. The coefficients of Variation (CV) for inter-assay analyses are $5.1 \%$ at 25 -hydroxyvitamin $\mathrm{D}$ of $47.8 \mathrm{nmol} / \mathrm{L}$ and $6.9 \%$ at 25 -hydroxyvitamin $\mathrm{D}$ of $83.0 \mathrm{nM}$. [8]

To prepare samples, $2 \mathrm{ml}$ of precipitation reagent with internal standard was dispensed into a disposable glass test tube. Then $1.0 \mathrm{ml}$ of serum (calibrator, control, or patient sample) was added to the tube without mixing of contents to avoid balling of the protein. The tube was allowed to sit for $5 \mathrm{~min}$ at room temperature, after which it was mixed in a vortex for 10 seconds to obtain a flocculent precipitate. After waiting for another $5 \mathrm{~min}$ the tube was again vortex-mixed and centrifuged at $2000 \mathrm{~g}$ for $10 \mathrm{~min}$. The clear supernatant was decanted into a disposable glass test tube which was protected from exposure to natural sunlight to prevent degradation of analytes. The unit sequentially was conditioned the Strata-X cartridge in the DEC zone with $2.0 \mathrm{ml}$ of $\mathrm{CH}_{3} \mathrm{CN}$ followed by $2.0 \mathrm{ml}$ of $35: 65 \mathrm{CH}_{3} \mathrm{CN}$-water. Then $1.0 \mathrm{ml}$ of water was added to each extract and transferred $3.5 \mathrm{ml}$ of extract mixture to the DEC. Then the DEC was rinsed with $2.0 \mathrm{ml}$ of 35:65 $\mathrm{CH}_{3} \mathrm{CN}$-water and was eluted the Strata-X cartridge in the DEC zone with $2.0 \mathrm{ml}$ of $\mathrm{CH}_{3} \mathrm{CN}$. The eluate was dried at $35^{\circ} \mathrm{C}$ under a stream of nitrogen and the dry extract was then reconstituted with $150 \mu \mathrm{l}$ of ethyl acetate- $\mathrm{CH}_{3} \mathrm{CN}$ (5:95 by volume) and vortex-mixed for 5 seconds. Water $(110 \mu \mathrm{L})$ was then added to the tube, and the contents were vortex mixed for 5 seconds. The sample was centrifuged at $2000 \mathrm{~g}$ for 10 min to settle the precipitate. The clear liquid was transferred to an amber coloured vial. The sample was finally capped. The extract was stable for 3 days at room temperature. The processor software calculated relative retention time for peak identification and peak-height ratio for quantification.

\subsubsection{Chemiluminiscent Immunoassay (CLIA)}

25-hydroxyvitamin $\mathrm{D}$ in serum was measured with automated chemiluminiscent immunoassay technology (VITROS eci, Johnson and Johnson Ortho Clinical Diagnostics). CV for inter-assay analyses is $5.8 \%$ at a 25 hydroxyvitamin D level of $39.5 \mathrm{nmol} / \mathrm{L}$ and $3.1 \%$ at $121.25 \mathrm{nmol} / \mathrm{L}[11]$.

\subsubsection{EIA}

Vitamin D of the subjects was also estimated using solid phase Enzyme Linked Immunosorbent Assay performed (EIA) on microtiterplates (DIAsource ImmunoAssays, Belgium). Within-run CV including extraction $6.8 \%$, and between-run CV 6.2\% [10].

\subsection{Statistical Analysis}

The data for biochemical analysis was subjected to standard statistical analysis using the Statistical Package for Social Science (SPSS) 11.5 software for windows.

\section{RESULT}

The personal profiles and clinical parameters of all the subjects under study are shown in Table 1.

Mean value of blood vitamin D obtained by HPLC method (69.34 nmole/L) are more close to CLIA (68.46 nmole/L) than EIA (67.18 nmole/L). Another observation is that concentration vitamin D in EIA has lower mean has wider SD and confidence interval than other two samples as shown in Table 2.

Table 1. Personal profiles of the subjects.

\begin{tabular}{cc}
\hline Age & $45.37 \pm 8.51$ \\
\hline Number of subjects & $\mathrm{n}=216$ \\
Sex: & 129 \\
Male & 87 \\
Female & \\
Demographic data: & 123 \\
Urban background & 93 \\
Rural background &
\end{tabular}

Value are mean $\pm \mathrm{SD} ; \mathrm{n}=$ number of cases.

Table 2. Comparison of mean value of three methods of blood vitamin $\mathrm{D}$ estimation.

\begin{tabular}{cc}
\hline $\begin{array}{c}\text { Methods of blood } \\
\text { vitamin D estimation }\end{array}$ & $\begin{array}{c}\text { Concentration of vitamin D } \\
\text { in blood (nmole/L) }\end{array}$ \\
\hline HPLC & $69.34 \pm 8.02$ \\
CLIA & $68.46 \pm 8.01$ \\
EIA & $67.18 \pm 8.21$ \\
HPLC vs CLIA & $\mathrm{CI}=0.6525-1.1068$ \\
HPLC vs EIA & $\mathrm{CI}=1.8916-2.4232$ \\
\hline
\end{tabular}

Values are mean $\pm \mathrm{SD} ; \mathrm{CI}=$ confidence interval. 
Regression analysis is performed to evaluate which method of vitamin D estimation and which method shows best correlate with HPLC. Over the whole range of vitamin $\mathrm{D}$ values in HPLC, the CLIA shows a higher $\mathrm{r}$ value $(r=0.978, p<0.0001)$ and EIA a lower $r$ value $(r$ $=0.970, \mathrm{p}<0.0001)$ of vitamin $\mathrm{D}$ of same individual. That signifies that values of vitamin D in CLIA of the subjects is more significantly correlated with value obtained from HPLC than EIA as shown in Table 3 and Figure 1.

The area under the curve (AUC) of ROC curve be-

Table 3. Pearson correlation of HPLC with CLIA and EIA.

\begin{tabular}{ccc}
\hline Category & r value & Significance \\
\hline HPLC vs CLIA & 0.978 & 0.000 \\
HPLC vs EIA & 0.970 & 0.000 \\
\hline
\end{tabular}

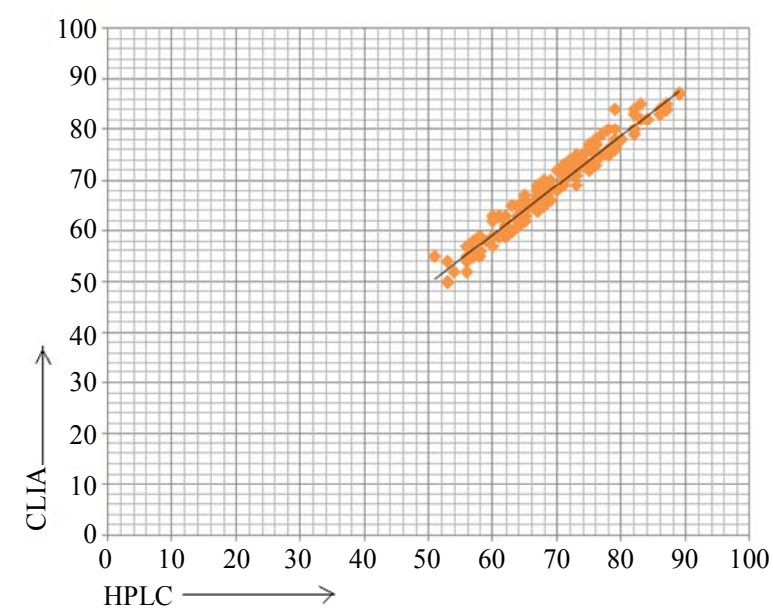

(a)

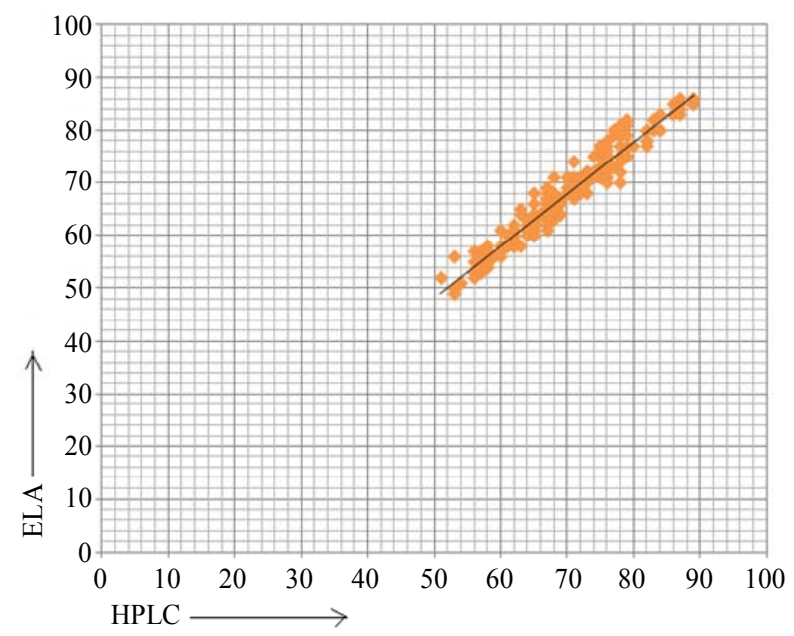

(b)

Figure 1. Scatter diagram (a) Correlation of vitamin D concentration between HPLC \& CLIA method (b) Correlation of vitamin D between HPLC \& EIA method. tween values of blood vitamin D obtained by HPLC and CLIA is more than between HPLC and EIA as shown in Table 4 and Figure 2.

\section{DISCUSSION}

Deficiency of vitamin D is common and represents a major health problem. Early in life, vitamin D deficiency causes growth retardation and rickets, whereas in adults it is well known to accelerate osteopenia and osteoporosis. Accumulating evidence also links a low vitamin D nutritional status to highly prevalent chronic illnesses, including common cancers, autoimmune diseases, infectious and cardiovascular diseases [12-14]. Rapid screening for vitamin $\mathrm{D}$ status is essential as it allows for monitoring a patient's response to vitamin D therapy and

Table 4. Area under the ROC curve and CI between the different methods of serum vitamin $\mathrm{D}$ estimation.

\begin{tabular}{ccc}
\hline Category & $\begin{array}{c}\text { Area under the } \\
\text { ROC curve }\end{array}$ & CI \\
\hline Between HPLC and CLIA & 0.578 & $0.524-0.632$ \\
Between HPLC and EIA & 0.534 & $0.48-0.589$ \\
\hline
\end{tabular}

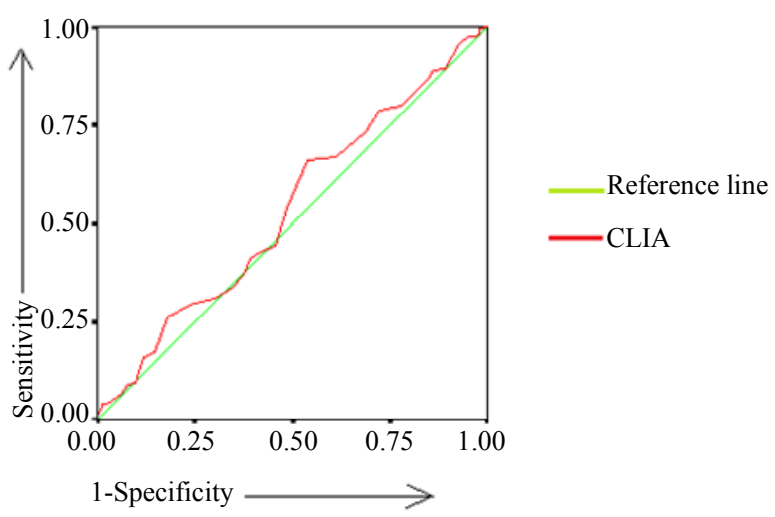

(a)

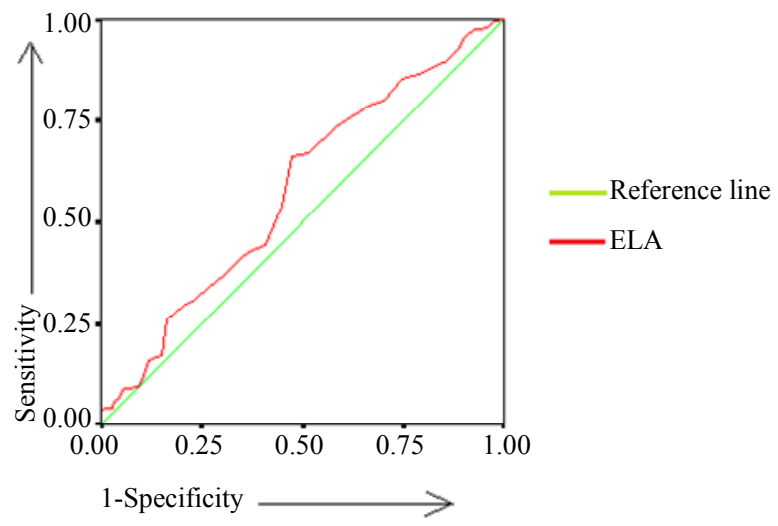

(b)

Figure 2. Receiver Operative Characteristic (ROC) curves of methods of serum vitamin D (a) HPLC and CLIA (b) HPLC and EIA. 
also evaluation of treatment effect [10] therefore, samples providing immediate and reliable results are highly desirable. Clinical laboratory scientists have a diverse array of vitamin $\mathrm{D}$ testing methods from which to choose. With the respect to vitamin $\mathrm{D}$ estimation chromatographic separation is suitable as a reference method 15 , since it is capable of resolving $\mathrm{D}_{2}$ and $\mathrm{D}_{3}$ forms as well as the $25 \mathrm{OH}, 1,25(\mathrm{OH})_{2}$ and $24,25(\mathrm{OH})_{2}$ metabolites. A C18 reverse phase column with isocratic or gradient elution using acetonitrile/water is now the standard procedure and diode array detection following hexane extraction gives results of sufficient clinical sensitivity [15]. Whilst the reference method is suitable for vitamin D estimation, their complexity and derivatisation requirements mitigate against regular use. More practical and easier alternatives method is CLIA or EIA [16]. The introduction of antibody preparations against vitamin D metabolites made it possible to develop assays that generally are more specific vitamin D assays, thus requiring fewer purification steps and thus reduces time (1). In present study, when cut-off values are used that are advocated in reagent manual, the value of vitamin $D$ of CLIA are more closed to the HPLC than value obtained by EIA. So, CLIA can replace the HPLC method which is too much lengthy. Previous study also gives support towards this factor due to easy performance, low cost and high sensitivity (1).

\section{CONCLUSION}

The study concluded that CLIA not the EIA could replace the HPLC for estimation of blood vitamin D effectively and can be used as a rapid screening test for vitamin D status and for monitoring a patient's response to vitamin D therapy for reasons of convenience, speed, turnaround time and cost.

\section{ACKNOWLEDGEMENTS}

Authors are thankful to Mr.Kamal Kumar Mukim, Director, Gautam Laboratories, Kolkata and Mr. Pinaki Karmakar, Technician, Department of Immunohematology, Gautam Laboratories, Kolkata for constant support \& inspiration.

\section{REFERENCES}

[1] Bischoff-Ferrari, H.A., Dietrich, T., Orav, E.J. and Dawson-Hughes, B. (2004) Positive association between 25hydroxy vitamin D levels and bone mineral density: A population-based study of younger and older adults. American Journal of Medicine, 116, 634-639. http://dx.doi.org/10.1016/j.amjmed.2003.12.029

[2] Lips, P. (2001) Vitamin D deficiency and secondary hyperparathyroidism in the elderly: Consequences for bone loss and fractures and therapeutic implications. Endocrine Reviews, 22, 477-501.

http://dx.doi.org/10.1210/er.22.4.477
[3] Bischoff-Ferrari, H.A., Dietrich, T., Orav, E.J., Hu, F.B., Zhang, Y., et al. (2004) Higher 25-hydroxyvitamin D concentrations are associated with better lower-extremity function in both active and inactive persons aged $>$ or $=$ $60 \mathrm{y}$. The American Journal of Clinical Nutrition, 80, 752-758

[4] Holick, M.F. (2004) Vitamin D: Importance in the prevention of cancers, type 1 diabetes, heart disease, and osteoporosis. The American Journal of Clinical Nutrition, 79, 362-371.

[5] Forman, J.P., Bischoff-Ferrari, H.A., Willett, W.C., Stampfer, M.J. and Curhan, G.C. (2005) Vitamin D intake and risk of incident hypertension: Results from three large prospective cohort studies. Hypertension, 46, 676-682. http://dx.doi.org/10.1161/01.HYP.0000182662.82666.37

[6] Hypponen, E., Laara, E., Reunanen, A., Jarvelin, M.R. and Virtanen, S.M. (2001) Intake of vitamin D and risk of type 1 diabetes: A birth-cohort study. Lancet, 358, 15001503. http://dx.doi.org/10.1016/S0140-6736(01)06580-1

[7] Melamed, M.L., Michos, E.D., Post, W. and Astor, B. (2008) 25-hydroxyvitamin D levels and the risk of mortality in the general population. Archives of Internal Medicine, 168, 1629-1637. http://dx.doi.org/10.1001/archinte.168.15.1629

[8] Tsugawa, N., Suhara, Y., Kamao, M. and Okano, T. (2005) Determination of 25-hydroxyvitamin D in human plasma using high-performance liquid chromatographytandem mass spectrometry. Analytical Chemistry, 77, 3001-3007. http://dx.doi.org/10.1021/ac048249c

[9] Hollis, B.W. (2004) Editorial: The determination of circulating 25-hydroxyvitamin D: No easy task. The Journal of Clinical Endocrinology \& Metabolism, 89, 3149-3151. http://dx.doi.org/10.1210/jc.2004-0682

[10] Lind, C., Chen, J. and Byrjalsen, I. (1997) Enzyme immunoassay for measuring 25-hydroxyvitamin D3 in serum. Clinical Chemistry, 43, 943-949.

[11] Aslan, K. and Geddes, C.D. (2009) Metal-enhanced chemiluminescence: Advanced chemiluminescence concepts for the 21st century. Chemical Society Reviews, 38, 25562564. http://dx.doi.org/10.1039/b807498b

[12] Bouillon, R., Bischoff-Ferrari, H. and Willett, W. (2008) Vitamin D and health: Perspectives from mice and man. Journal of Bone and Mineral Research, 23, 974-979. http://dx.doi.org/10.1359/jbmr.080420

[13] Bouillon, R., Carmeliet, G., Verlinden, L., et al. (2008) Vitamin D and human health: Lessons from vitamin D receptor null mice. Endocrine Reviews, 29, 726-776. http://dx.doi.org/10.1210/er.2008-0004

[14] Holick, M.F. (2007) Vitamin D deficiency. The New England Journal of Medicine, 357, 266-281. http://dx.doi.org/10.1056/NEJMra070553

[15] Turpeinen, U., Hohenthal, U. and Stenman, U.H. (2003) Determination of 25-hydroxyvitamin $\mathrm{D}$ in serum by HPLC and immunoassay. Clinical Chemistry, 49, 15211524. http://dx.doi.org/10.1373/49.9.1521

[16] Wootton, A.M. (2005) Improving the measurement of 25-hydroxyvitamin D. The Clinical Biochemist Reviews, 26, 33-36. 